\title{
Mobility Management using the IP Protocol
}

\author{
Imtiaz A. Halepoto ${ }^{1}$, Adnan Manzoor ${ }^{2}$, Nazar H. Phulpoto ${ }^{2}$, Sohail A. Memon ${ }^{3}$, Muzamil Hussain ${ }^{4}$ \\ ${ }^{1}$ Department of Computer Systems Engineering, QUEST Nawabshah, Pakistan \\ ${ }^{2}$ Department of Information Technology, QUEST Nawabshah, Pakistan \\ ${ }^{3}$ Department of Mathematics, SALU Khairpur, Pakistan \\ ${ }^{4}$ Department of Computer Science, QUEST Nawabshah, Pakistan
}

\begin{abstract}
Time critical applications, such as VoIP and video conferencing require Internet connectivity all of the time for better performance. Moreover, in case of vehicular networks, it is very common for mobile devices to move from one network to another. In such scenarios the sudden changes in the network connectivity may cause problems, which affects the data transmission rate. The movement of a mobile node from one network to another is also a challenge for the routers to maintain the routing information as well as to forward the data to the corresponding node. In all of the aforementioned scenarios, the switching between the networks with minimum latency improves the performance, i.e. in terms of mobility and availability of the network. The Mobile IP protocol serves the purpose of seamless handover of mobile devices from one network to another. A mobile node maintains its permanent IP address using the Mobile IP protocol while moving to a foreign network. When a mobile node establishes the connection with the foreign network the data packets transmitted from the home network are redirected to the foreign network. The Mobile IP protocol establishes a tunnel between the home network and the foreign network. The process of tunneling continues until the mobile node moves back to the home network or when the foreign network advertises the new IP address of the mobile node. With the increasing number of wireless devices the mobility is the key challenge. The devices with multiple interfaces such as mobile phone which uses 4G as well as WiFi, the urge for the availability of the Internet is also high. This paper provides a deep discussion about the Mobile IP protocol and its implementation. A network scenario is proposed with the configuration of the Mobile IP. According to the obtained results of the simulations, the Mobile IP protocol increases the availability of the network connection as well as it achieves the larger throughput when compared with the scenario without using the Mobile IP.
\end{abstract}

Keywords-Mobility; Mobile IP; 4G; foreign network; permanent IP

\section{INTRODUCTION}

The main protocol that is most widely used over the Internet is TCP [1]. However, TCP needs the services of the network layer protocols. The famous network layer protocol is the IP protocol. At the time of IP proposal, it was intended for the stationary networks with a constant IP address assigned to each host. With the recent advances in the technology like ubiquitous computing and pervasive computing the mobility of devices while maintaining the Internet connection have become the normal trend. As the IP is mainly for stationary devices so in case of mobility [12] when a device wanders far, it disconnect with the home network. For example, when a device is outside wireless range of the service provider. In case of cellular networks for example 4G [11], the technique like link layer and roaming minimize the handover [2]. However, many of of companies still use the mobile IP. In many cases some of the handover management [16] schemes are used to reduce the transmission latency when a device travels from the home network to a foreign network. However, the latency in the handover management schemes is not according the expectations and also raise many questions regarding the availability of the network.

In order to cope with mobility and the availability of the Internet (or network connection) some of the protocols are proposed at the transport layer, such as the stream control transmission protocol (SCTP). With SCTP, it is possible to remain connected with multiple networks at the same time. SCTP increases the Internet availability as well as provides features to enhance the mobility. In order to facilitate the mobility, SCTP uses the multihoming technique [3], which provides the ability to a device to remain connected with multiple networks (i.e. home and foreign agents). However, there are many issues in SCTP such as congestion control, flow control and scheduling of data transmission [4]-[7]. In Fig. 1, a network is shown with two multihomed devices. Device A with two interface and device B with three interfaces connected by using the SCTP protocol. The other solution to the mobility and the availability is the use of Mobile IP protocol [8]-[10]. The assortment of Mobile IP with the other mobility techniques is beneficial. With Mobile IP it is allowed to devices to use the permanent IP address while moving to a foreign network. This protocol maintains the Internet connectivity and improves the transmission rate in the scenarios of voice over IP (VoIP), virtual private networks, video streaming and in wireless sensor networks.

The paper provides the details of the implementation of Mobile IP protocol in NS2 [13]. The simulation are performed on network where nodes as stationary and mobile. The result are collected while using the Mobile IP protocol and are compared with the same scenario that does not uses the Mobile IP protocol. A network is proposed to observe and analyze the importance of Mobile IP with the following scenarios:

- The movement of a mobile host from one network another network.

- Transmission of data from a wired device to a wireless mobile node.

- The movement of two mobile nodes moving to a foreign network while receiving data from a fixed node.

- the movement of two mobile nodes to a foreign network while sending data each other.

In the rest of the paper, the details of the Mobile IP are 


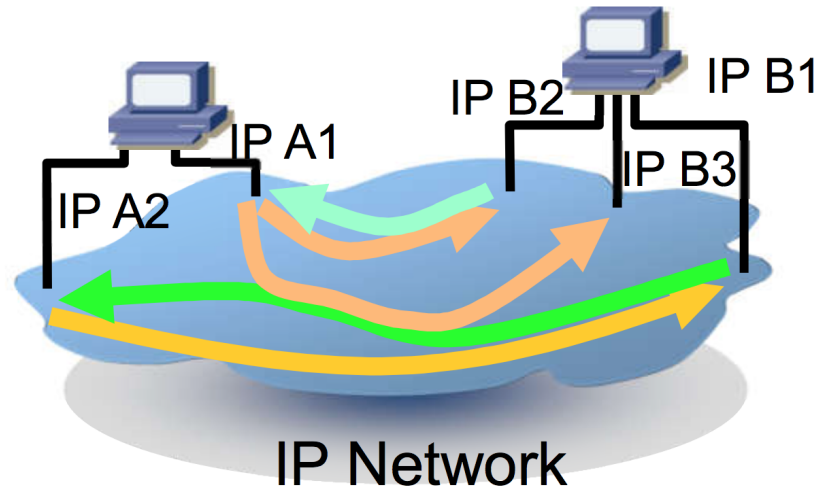

Fig. 1. SCTP multihoming.

presented in Section II. The creation of network scenario and implementation details of mobile IP are described in Section III. The discussion on the observed results are presented in Section IV. The conclusion and recommendations for future are presented in Section V.

\section{Mobility at Network LAyER}

The Internet protocol that provides seamless connectivity while maintaining the IP address is called the Mobile IP protocol. It is standardized in IETF RFC 5944. The trend of wireless and mobile communication is increasing, the device with multiple interfaces, such as mobile phone are also large in number. Many of the service providers of $4 \mathrm{G}$ has incorporated new techniques for the mobility management of the mobile phones. The implementation of mobility management techniques in cellular technology uses the link layer methods, however such methods face many problems specifically in security and trustworthy. Mobile IP is an alternative to such link layer techniques. It provides the location independent routing over the Internet. Each device using the Mobile IP maintains a permanent IP address. When a device travels to a foreign network, it creates a tunnel which redirects the packets from belong the mobile host in home network to the mobile host in foreign network.

While using the Mobile IP, a host maintain two kinds of addresses. First, the home address, it assigned to all of the devices connected to the home network. Second, the care of address, it is the address of a mobile host in the foreign network. Two kids of agents are also used for the communication purpose. These agents serve as the routers and help in data forwarding. They also maintain a data structure to maintain the IP information for data forwarding. One agent is called the home agent (HA), it stores the permanent IP address when a mobile host is in the home network. Other is called the foreign agent (FA), it advertises the care of address of a mobile host when in the foreign network. The working of these agents is as a three step process, these are agent discovery, agent advertisement and agent solicitation. The key functionality of these agents is as follows:

- Agent discovery is a method used by the mobile host to discover the available agents in the area.

- Agent advertisement is done by the agents through

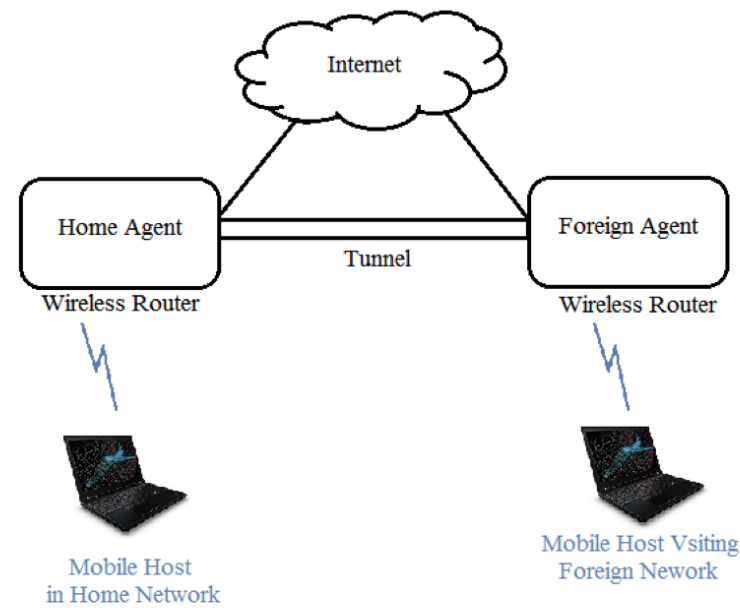

Fig. 2. The concept of Mobile IP.

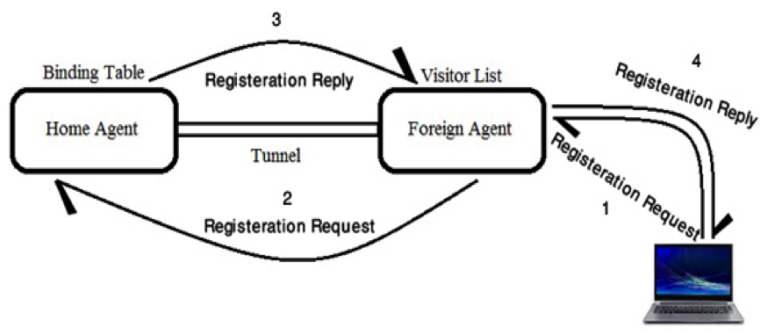

Fig. 3. Registration process in Mobile IP.

the ICMP (Internet message control protocol) to show their presence and availability.

- Agent solicitation messages are used by the mobile hosts to ask for the advertisement from the agents. Once the mobile receives the advertisement, it registers itself with the foreign agent, which forwards information of care of address to the home agent.

A mobile host using Mobile IP is also allowed to register with more than one foreign agents. When the mobile node returns to the home network it de-register itself from other networks. Due to the wireless communication, the Mobile IP face challenges in providing authentication. At the time of registration, the authentication is very critical, Mobile IP uses MD5 (128bits) algorithm to minimize the issue. This algorithm also helps in minimizing the denial of service attack, where an attacker sends a large number of bogus registration requests (see, Fig. 2 and 3).

\section{MOBILE IP IMPLEMENTATION AND CONFIGURATION}

The simulation scenario consist of wired and wireless nodes as shown in Fig. 4. Topology implementation is taken from the Marc Greis in [14], however the node movements are configured to simulate the two mobile hosts to send and receive data to each other. Number of wired nodes are 2. Number 


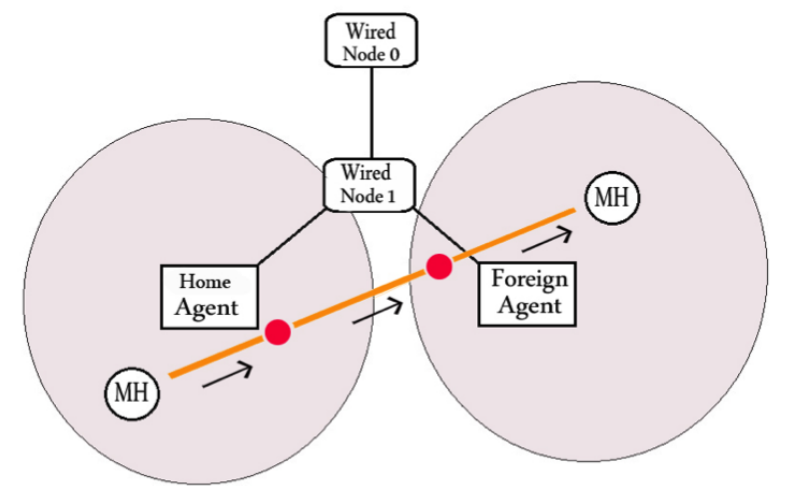

Fig. 4. Network topology.

of mobile nodes are 2. Number of base station nodes are 2. Channel type used is wireless. Propagation model is configured to two-way. Antenna type is omni-antenna. Link Layer type is LL. Packet length is set to 50. Routing Protocol used at the network layer is DSDV (Destination sequenced distance vector). Dimensions of topology are $x=y=670$. The total simulation time is 250 seconds. The simulations are carried out in NS2 and results are collected on the basis of average of 20 experiments for each of the simulation scenario. All other parameters of the network layer are set to default values. On the transport layer, TCP protocol is configured. TCP agents are defined as the source and the destination. For example, in first scenario the source is wired node and the destination is mobile host.

After the topology is designed, a simple scenario of two simple wired nodes w0 \& w1, which are then connected to each other using $5 \mathrm{mbps}$ link. Then two base station are added, which are named as Home Agent (HA) and Foreign Agent (FA), which are connected to the wired node w1 using $5 \mathrm{mbps}$ link. A mobile host is defined to move in between HA and FA. In first movement the mobile host stays remain in the range of Home Network and after 100 seconds it starts travel to the foreign agent. Then after 200 seconds it travels back to the Home Network. From the operating system side, Ubuntu 14.04 is used on the virtual machine. AWK scripting is used to calculate the throughput [15]. Three kinds of simulations are performed by changing the movement of mobile nodes:

1) The mobile host moves from the HA to the FA and then back to the area of HA. The data transmitter is w0 and the receiver is the mobile host.

2) In this scenario, two mobile hosts are configured. One is in the HA and one is in the FA. The sender is w0 and two mobile hosts are the receiver.

3) In this scenario, two mobile hosts send and receive the data to each other as shown in Fig. 4.

In all of the simulations, Mobile IP simulation is compared with the same scenario without the Mobile IP.

\section{RESUlTS AND Discussions}

\section{A. One Mobile Host}

Two scenarios using one with Mobile IP protocol and other without using Mobile IP protocol are evaluated. In both scenarios, the start time is 0 and the end time 250seconds. Mobile host from 0s to 100s remains in the range of Home Network. After 100s Mobile Host travels towards the Foreign Network and stays there until 200s. After 200s it travels back to the Home Network.

Fig. 5 clearly shows that from time about 0 s to $110 \mathrm{~s}$ in both cases the throughput is about $0.7 \mathrm{Mbps}$. After $110 \mathrm{~s}$ the graph suddenly goes down because mobile host in both scenarios are disconnected because mobile host is neither in the range of the home network nor in the range of the foreign network. After some time about 160s Mobile Host using Mobile IP is connected and registered to the foreign network by that data transmission resumes and throughput goes up again about to $0.7 \mathrm{Mbps}$. One other hand in the same scenario but mobile host without mobile IP support remains disconnected. When the time slot in graph reaches to 210 s throughput goes down due to the coordinates of mobile host, i.e. outside home agent and foreign agent. It remains disconnected until 240s where the mobile host in both scenarios are back to the network and in the graph it is represented with a throughput value of $0.7 \mathrm{Mbps}$. In Fig. 4, from 110 s to 240 s, it clear that the mobile host remains disconnected when no Mobile IP protocol is used. It is represented by the red line. However, with the use of Mobile IP protocol the mobile host resumes the connection at around time 160 s to time 210 s while maintaining the connection with the foreign agent. The throughput with and without using the Mobile IP in this scenario are $59.80 \mathrm{kbps}$ and $42.81 \mathrm{kbps}$.

\section{B. Two Mobile Hosts}

In this scenario two Mobile Hosts are used. The mobile hosts belong to different networks. One mobile host uses home agent HA. Other uses the home agent FA. The simulation is repeated two times, i.e. with and without using the Mobile IP protocol.

Fig. 6 graph shows the performance of Mobile IP when two mobile hosts are used. Mobile node with and without Mobile IP part of HA. In both cases MH uses home access. Throughput rise about $0.7 \mathrm{Mbps}$. After 110s throughput goes OMbps. Reason is that mobile node in both conditions are out of reach of home agent and foreign agent. $\mathrm{MH}$ configured to Mobile IP regaining throughput at about $155 \mathrm{~s}$. MH configured to Mobile IP have throughput about $0.7 \mathrm{Mbps}$. $\mathrm{MH}$ without Mobile IP is down because of unknown to foreign network. After 210 s both are returning to home. Unavailable throughput for interval of time due to out of range to HA \& FA. Reaching back at home location the both lines up to throughput is available on the time slot 240 s goes to $0.7 \mathrm{Mbps}$. It is shown in Fig. 6 that with Mobile IP, the mobile host connects to the foreign agent at approximately time 160s and remains connected up to time 210s. Similarly, in Fig. 7, the seconds mobile host resumes connection from approximately $145 \mathrm{~s}$ to 210 s as represented by red line.

\section{Mobile Sender and Mobile Receiver}

In third scenario, the role of sending and receiving is assigned to the mobile nodes. The mobile node 1 and 2 are configured to send and receive data from each other. The mobile node 1 starts from the HA and mobile node 2 starts from the FA as shown in Fig. 4. 
TABLE I. PARAMETERS AND VALUES

\begin{tabular}{|lllll|}
\hline Scenario & Average Throughput (kbps) & Packets Sent & Packets Received & End-to-end Delay \\
\hline 1. & & & & \\
\hline Mobile IP & 59.80 & 15290 & 14950 & 106795.95 \\
\hline Without Mobile IP & 42.81 & 11601 & 10703 & 76346.42 \\
\hline 2. & & & & 1057115.27 \\
\hline Mobile IP & 55.99 & 13868 & 13541 & 80811.15 \\
\hline Without Mobile IP & 42.29 & 10927 & & 112724.19 \\
\hline 3. & & & 15721 & 58487.06 \\
\hline Mobile IP & 64.78 & 16037 & 9166 & 89640.36 \\
\hline Without Mobile IP & 36.74 & 9511 & & 59423.14 \\
\hline 4. & & & 24573 & 27455 \\
\hline Mobile IP(HA-FA) & 98.46 & 27576 & & \\
\hline Mobile IP(FA-HA) & 110.01 & & & \\
\hline
\end{tabular}

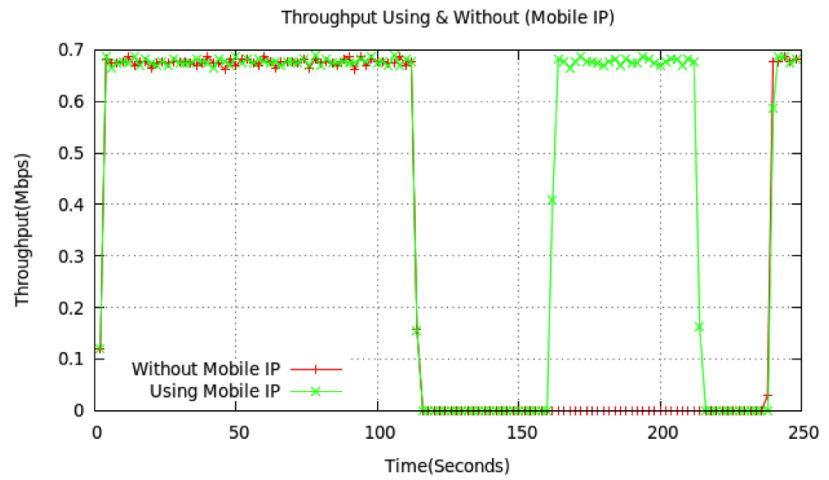

Fig. 5. Experiment 1: One mobile node.

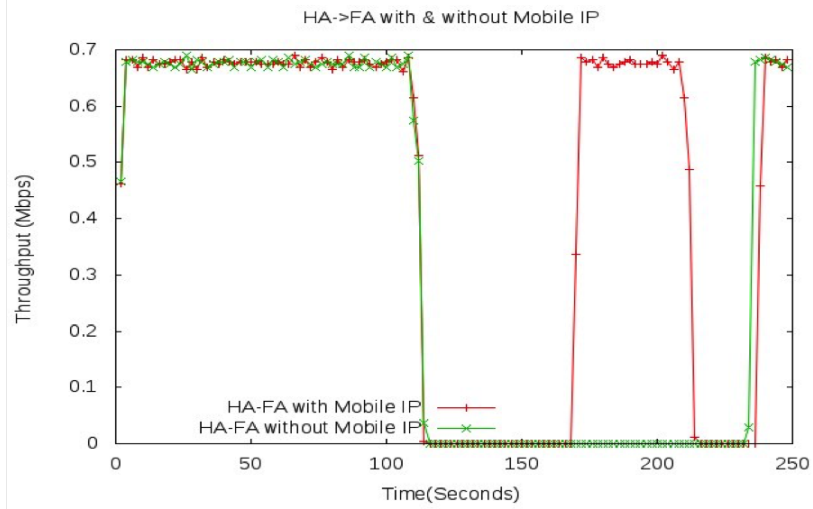

Fig. 6. Experiment 2.1: Two mobile node.

Fig. 8 resultant graph showing that from time 0 s the both mobile hosts have available throughput is about $0.7 \mathrm{Mbps}$ until the time 110s and throughput goes $0 \mathrm{Mbps}$ because both mobile goes out of reach of home agent and mobile agent. And both agent again available for throughput for few second and disconnect. Mobile Host of FA is again regaining throughput at about 145s and mobile host of HA is remain unconnected and will throughput available at 160s. Both hosts connectivity

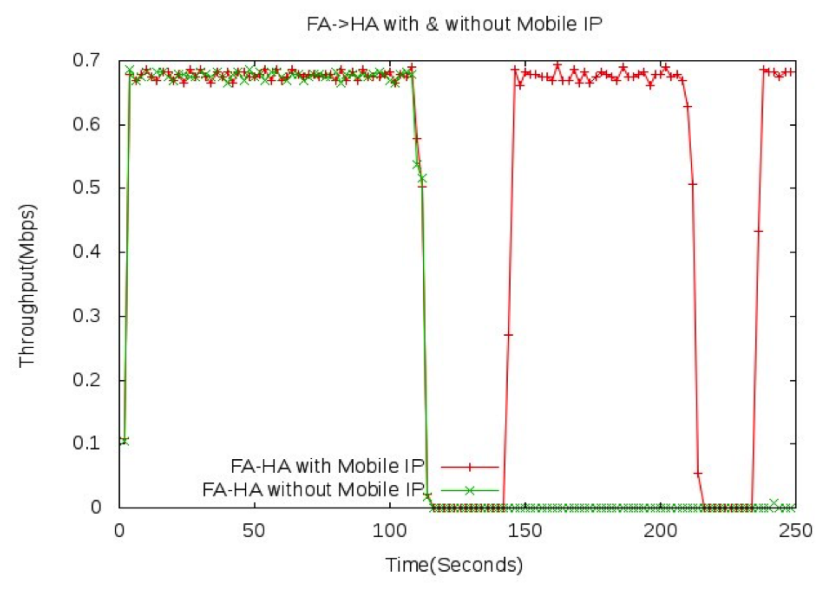

Fig. 7. Experiment 2.2: Two mobile node.

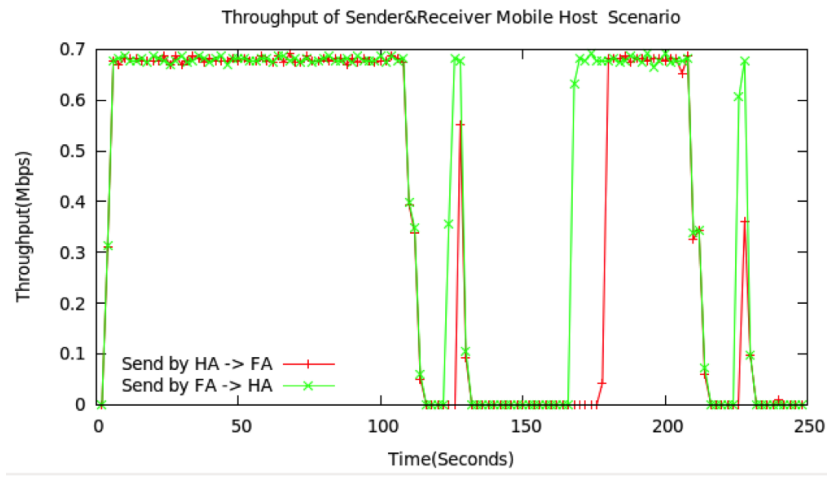

Fig. 8. Experiment 3: Two sending receiving mobile nodes.

is maintained with $0.7 \mathrm{Mbps}$ until $210 \mathrm{~s}$ after that gain mobile agents are back to the home location remain unconnected until they are in range and throughput for both is available on the time slot $240 \mathrm{~s}$ at about $0.7 \mathrm{Mbps}$. The overall result of this experiments shows that the increased mobility while using the Mobile IP. The packets sent and received and the throughput 
of both mobile node 1 and 2 are depicted in Table I.

\section{CONCLUSION AND RECOMMENDATIONS FOR FURTHER STUDY}

With the rapid growth in the mobile applications for video streaming and conferencing, the most important is the availability of the Internet connection. The availability of the network connection is related with the mobility, as the mobile devices move from one place to other and join the foreign networks. The transport layer also provide the solutions to the availability and mobility with the help of multi-interface devices. Where, a device maintains the Internet connection through a number of network interface cards. One of the simplest solution to the mobility and the availability of the Internet is the use of Mobile IP protocol at the network layer. Mobile IP uses tunneling concept, that allows a mobile device to use the same IP address in a remote or foreign network. Tunneling redirects the packets of mobile host to the foreign network. This paper provides a detailed study of Mobile IP protocol. The protocol is implemented and tested against the traditional network that do not support mobility. The evaluation shows that the Mobile IP increases the network availability and mobility. By that, it also improves the data transmission rate. In future, the concept of Mobile IP with other mobility management schemes such as handover should be considered. The evaluation of Mobile IP concept with the IPv6 would be good research.

\section{REFERENCES}

[1] Allman, Mark, Vern Paxson, and Ethan Blanton. TCP congestion control. No. RFC 5681. 2009.

[2] Borella, Michael S. "System and method for control of packet data serving node selection in a mobile internet protocol network." U.S. Patent No. 7,346,684. 18 Mar. 2008.
[3] Ishakian, Vatche, Joseph Akinwumi, Flavio Esposito, and Ibrahim Matta. "On supporting mobility and multihoming in recursive internet architectures." Computer Communications 35, no. 13 (2012): 1561-1573

[4] Halepoto, Imtiaz Ali. "Scheduling and flow control in CMT-SCTP." HKU Theses Online (HKUTO) (2014).

[5] Halepoto, Imtiaz A., Francis CM Lau, and Zhixiong Niu. "Scheduling over dissimilar paths using CMT-SCTP." Ubiquitous and Future Networks (ICUFN), 2015 Seventh International Conference on. IEEE, 2015.

[6] BHANGWAR, Noor H., Imtiaz A. HALEPOTO, Intesab H. SADHAYO, Suhail KHOKHAR, and Asif A. LAGHARI. "On Routing Protocols for High Performance." Studies in Informatics and Control 26, no. 4: 441448, 2017.

[7] Halepoto, Imtiaz A., Francis CM Lau, and Zhixiong Niu. "Management of buffer space for the concurrent multipath transfer over dissimilar paths." Digital Information, Networking, and Wireless Communications (DINWC), 2015 Third International Conference on. IEEE, 2015.

[8] Perkins, Charles E. "IP mobility support for IPv4, revised." (2010).

[9] Jung, J-W., et al. "Performance evaluation of two layered mobility management using mobile IP and session initiation protocol." Global Telecommunications Conference, 2003. GLOBECOM'03. IEEE. Vol. 3. IEEE, 2003.

[10] Jnsson, Ulf, et al. "MIPMANET: mobile IP for mobile ad hoc networks." Proceedings of the 1st ACM international symposium on Mobile ad hoc networking \& computing. IEEE Press, 2000.

[11] Frattasi, Simone, et al. "Defining $4 \mathrm{G}$ technology from the users perspective." IEEE network 20.1 (2006): 35-41.

[12] Van de Groenendaal, Johan, and Amitava Chakraborty. "Mobility management in wireless networks.” U.S. Patent No. 7,634,252. 15 Dec. 2009.

[13] Issariyakul, Teerawat, and Ekram Hossain. Introduction to network simulator NS2. Springer Science \& Business Media, 2011.

[14] Greis, Marc. "Marc Greis tutorial for the ucb/lbnl/vint network simulator ns." (2004).

[15] Aho, Alfred V., Brian W. Kernighan, and Peter J. Weinberger. The AWK programming language. Addison-Wesley Longman Publishing Co., Inc., 1987.

[16] Tsang, Ken CK, Cho-Li Wang, and Francis CM Lau. "Handoff performance comparison of mobile IP, fast handoff and mSCTP in mobile wireless networks." Parallel Architectures, Algorithms, and Networks, 2008. I-SPAN 2008. International Symposium on. IEEE, 2008 